\title{
Unconventional mass enhancement around the Dirac nodal loop in $\mathrm{ZrSiS}$
}

\author{
S. Pezzini,,${ }^{1,2}$ M. R. van Delft, ${ }^{1,2}$ L. Schoop, ${ }^{3}$ B. Lotsch, ${ }^{3}$ A. Carrington, ${ }^{4}$ \\ M. I. Katsnelson, ${ }^{2}$ N. E. Hussey, ${ }^{1,2, *}$ and S. Wiedmann ${ }^{1,2,+}$ \\ ${ }^{1}$ High Field Magnet Laboratory (HFML-EMFL), Radboud University, \\ Toernooiveld 7, Nijmegen 6525 ED, Netherlands. \\ ${ }^{2}$ Radboud University, Institute for Molecules and Materials, Nijmegen 6525 AJ, Netherlands. \\ ${ }^{3}$ Max Planck Institute for Solid State Research, \\ Heisenbergstr. 1, 70569 Stuttgart, Germany. \\ ${ }^{4}$ H. H. Wills Physics Laboratory, University of Bristol, \\ Tyndall Avenue, Bristol BS8 1TL, UK.
}

(Dated: August 7, 2018)

\begin{abstract}
The topological properties of fermions arise from their low-energy Dirac-like band dispersion and associated chiralities. Initially confined to points, extensions of the Dirac dispersion to lines and even loops have now been uncovered and semimetals hosting such features have been identified. However, experimental evidence for the enhanced correlation effects predicted to occur in these topological semimetals has been lacking. Here, we report a quantum oscillation study of the nodal loop semimetal $\mathrm{ZrSiS}$ in high magnetic fields that reveals significant enhancement in the effective mass of the quasiparticles residing near the nodal loop. Above a threshold field, magnetic breakdown occurs across gaps in the loop structure with orbits that enclose different windings around its vertices, each winding accompanied by an additional $\pi$ Berry phase. The amplitudes of these breakdown orbits exhibit an anomalous temperature dependence. These findings demonstrate the emergence of novel, correlation-driven physics in ZrSiS associated with the Dirac-like quasiparticles.
\end{abstract}


Condensed-matter systems with an energy-momentum dispersion topologically distinct with respect to (i.e. not adiabatically deformable into) the one of standard metals, semiconductors or insulators, represent a new exciting frontier in physics [1 5]. These materials host, in their bulk and/or surfaces, low-energy excitations mimicking relativistic particles, which opens new possibilities for the simulation of long-sought phenomena of high-energy physics, as well as for the realization of novel (quantum) information schemes. Graphene, topological insulators (TIs) and, more recently, Dirac and Weyl semimetals (WSMs) have been pioneering platforms for this research area [6, 7].

The advent of nodal-line semi-metals (NLSMs) [8 12], i.e. systems in which the conduction and valence bands cross each other along a closed trajectory (line or loop) inside the Brillouin zone, has opened up a new arena for the exploration of topological aspects of condensed matter that may be distinct from those associated with TIs or WSMs [13, 14]. It is claimed, for example, that due to the vanishing density of states near the Fermi level $\epsilon_{F}$, screening of the Coulomb interaction may become weaker in NLSMs than in conventional metals and remain long-ranged [15]. This, coupled with their metallic nature, could make NLSMs more susceptible to various types of order (e.g. superconductivity, magnetism or charge order), both in the bulk [16] and on the surface [17].

While the degeneracy points in WSMs are robust against any perturbation that preserves translational symmetry, nodal lines or nodal loops require additional crystalline symmetries, such as mirror or non-symmorphic symmetry, to protect the extended line-like touching between the conduction and valence bands [7, 14]. To date, only a few candidate NLSMs have been identified experimentally. In non-centrosymmetric $\mathrm{PbTaSe}_{2}$, strong spin-orbit coupling leads to the creation of nodal rings which have been confirmed by angle-resolved photoemission spectroscopy (ARPES) [10] while in $\mathrm{PtSn}_{4}$, ARPES has revealed a Dirac nodal arc structure that has been attributed to surface states [9]. In both systems, however, the topological elements of the electronic structure coexist with several other bands that cross the Fermi level $\epsilon_{F}$.

The third experimental class to have been identified thus far is $\operatorname{ZrSiX}(\mathrm{X}=\mathrm{S}, \mathrm{Se}, \mathrm{Te})$. The first of these, ZrSiS, has a number of distinct features. Firstly, it contains a diamondshaped Fermi surface (FS) close to a line of Dirac nodes that in contrast to $\mathrm{PbTaSe}_{2}$ and $\mathrm{PtSn}_{4}$, is the only band feature near $\epsilon_{F}$. Thus, the physical behavior of ZrSiS, be it bulkor surface-derived, is governed essentially by the electronic states in close proximity to 
the nodes. Secondly, the linear dispersion of these bands extends, in some regions of the Brillouin zone, over an energy range $(2 \mathrm{eV})$ that is much larger than is found in other Diraclike compounds. The range in which all bands are linearly dispersing is $\sim 0.5 \mathrm{eV}$. Spin-orbit coupling introduces a small gap $(\sim 0.02 \mathrm{eV})$ in the Dirac spectrum (as illustrated by the energy contour plot shown in Fig. S1 of the Supplementary Information).

ARPES studies have confirmed the existence of the diamond-shaped FS in ZrSiS, within the $\left(k_{x}, k_{y}\right)$ plane, in addition to a Dirac-like dispersion that extends over an energy range exceeding $1 \mathrm{eV}$ [11, 12]. Previous quantum oscillation studies have also reported evidence for pockets associated with the Dirac nodal loop in ZrSiS [18 21], ZrSiSe and ZrSiTe [22, 23]. In each case, the topological character of these loops was inferred from the observation of a phase shift in the quantum oscillations associated with the Berry phase of the orbit.

While the topological nature of NLSMs appears to be established, evidence for enhanced many-body effects, e.g. as a precursor to any new broken symmetry phases, has yet to emerge. Here, we report a magnetoresistance study of high-quality ZrSiS single crystals up to 33 Tesla. By extending the field range of earlier studies, we are able to resolve many new Shubnikov-de Haas ( $\mathrm{SdH}$ ) oscillation frequencies at all temperatures below $60 \mathrm{~K}$, including some very high frequency oscillations that arise due to magnetic breakdown across gaps along the nodal loop. The effective masses associated with these new frequencies are found to be significantly enhanced over conventional band-structure estimates. Moreover, the oscillation amplitude for the breakdown orbits exhibits an anomalous temperature dependence reminiscent of that recently found in the candidate topological Kondo insulator $\mathrm{SmB}_{6}$ [24]. Collectively, these results provide hints that ZrSiS lies close to a quantum phase transition,

possibly to some form of density wave order, and is thus an ideal material platform on which to explore novel correlation effects in topological matter.

\section{DIRAC NODAL LOOP}

The crystal structure of ZrSiS (tetragonal space group $P_{4} / \mathrm{nmm}$ ) is displayed in Fig. 17. ZrSiS has the PbFCl-type structure (like LiFeAs) [25, 26] with layers of Zr and S that are sandwiched between Si square nets extending in the ab-plane. The electronic band structure of bulk ZrSiS is shown in Fig. 1 p. The most dominant and noteworthy feature of the electronic structure is the series of linearly-dispersing bands that cross very close to $\epsilon_{F}$, 
giving rise to a nodal loop whose location is indicated in the corresponding Fermi surface plot shown in Fig. 1 1 . The final assembly of loops gives rise to a diamond-shaped Fermi surface within the $\left(k_{x}, k_{y}\right)$ plane that is quasi-two-dimensional yet strongly dispersive along $k_{z}$. Significantly, as indicated in Fig. 1 1 , the more parabolic bands (mostly originating from the sulfur states) are located far from the Fermi level, with the result that the Fermi surface depicted in Fig. 15 is composed uniquely from the almost-linearly dispersing bands.

\section{OSCILLATORY MAGNETORESISTANCE}

Figure $2 \mathrm{a}$ shows a series of magnetoresistance (MR) sweeps up to $33 \mathrm{~T}$ carried out on a $\mathrm{ZrSiS}$ single crystal at regular temperature intervals between $1.5 \mathrm{~K}$ and $60 \mathrm{~K}$ with the magnetic field applied perpendicular to the $a b$-plane. Very similar results obtained on a second crystal are presented in Fig. S2 of the Supplementary Information. As reported previously [18, 19, 21, 27], the MR is extremely large, reaching values of order $10^{4} \%$ in 33 $\mathrm{T}$ for $\mathbf{B} \| c$, and $7 \times 10^{5} \%$ with the field oriented at a polar angle $\theta=45^{\circ}$. Superimposed on top of the MR background are multiple SdH oscillations, some of which are very fast, as emphasized in the blow-up of the highest field data plotted in the right panel of Fig. 2 a .

A fast Fourier transform (FFT) of the lowest temperature sweep in Fig. 2a is shown in Fig. 2b. The oscillations can be readily split into two groups; a low frequency $(F<1 \mathrm{kT})$ and a high frequency $(F>7.5 \mathrm{kT})$ group. The low frequency spectrum is dominated by two peaks. The one at $240 \pm 5 \mathrm{~T}$ has been observed by a number of different groups [19 21] and corresponds to the $\alpha$ hole pocket (the 'petal'), highlighted in blue in the left panel of Fig. 2r, that is located at the vertex of the diamond. The second peak with a frequency of $600 \pm 10 \mathrm{~T}$ has not been reported previously. It is consistent (albeit $20 \%$ higher) than band structure estimates for the elongated $\beta$ electron pocket (the 'dog-bone') that runs parallel to the top rung of the nodal loop (see Fig. (1) $)$.

The higher frequency spectrum comprises a series of peaks ranging from $7.5 \mathrm{kT}$ to around $11 \mathrm{kT}$. These peaks do not correspond to any closed contour of the Fermi surface of ZrSiS shown in Fig. 1: but rather likely originate from magnetic breakdown orbits that encircle the diamond. This is confirmed by a number of observations. Firstly, as indicated by the dashed lines in the right panel of Fig. 22k, the lower set of five peaks are separated by units of $240 \mathrm{~T}$, i.e. by the frequency of the petal orbit. The magnitude of the peaks corresponds 
to a series of breakdown orbits (labelled $A \pm n \alpha$, where $n$ is an integer) that follow the inner surface of the dog-bones with each successive peak in the FFT spectrum incorporating one additional petal. A similar assignment can be made for the second set of peaks (labelled $B \pm n \alpha)$ that trace the outer surface of the dog-bones. Fig. 2 2 illustrates some of the possible breakdown combinations.

The second telling observation is the rapid suppression of the high frequency oscillations by a small tilt of the magnetic field away from the $c$-axis. Fig. 2rk contains a set of MR sweeps obtained at different polar angles, while Fig. 2 d shows the corresponding high frequency part of the FFT spectrum for increasing $\theta$. The oscillations (and the amplitude of the corresponding peaks) are found to be completely suppressed once $\theta>\theta_{c}$, with $2.2^{\circ}<\theta_{c}<$ $3.5^{\circ}$. This sudden collapse of the fast oscillations with small tilt angle is another clear indication of magnetic breakdown. As illustrated in Fig. 22f, some of the breakdown gaps must increase in size as the field is tilted. Since the tunneling probability drops exponentially with gap magnitude (or equivalently, the distance in $k$-space between adjacent elements of the breakdown orbit), the oscillation amplitude is then rapidly suppressed. A calculation of the attenuation in oscillation amplitude, plotted as a dashed line in Fig. 2rb, confirms this (details of the calculation itself can be found in the Supplementary Information).

\section{TOPOLOGICAL CHARACTER}

Having established that the different elements of the Fermi surface are tied to the nodal loop, we now turn to examine its topological character. According to the band structure calculations, the petal is located at the apex of the diamond and thus encircles a Dirac cone (see Fig. S1 of the Supplementary Information for a visualization of this). As a consequence, electrons performing any closed circuit including a petal should acquire a pi Berry phase [19]. Given that the breakdown orbits of the type $A+n \alpha$ and $B+n \beta$ are closed trajectories that differ by an integer number of petals (see Fig. $2 飞$ ), one expects there to be a relative phase shift of $\pi$ between orbits with $n$ even or odd. This phase shift should then be visible in the quantum oscillation trace. Figure 3 shows one such trace (black line, after subtraction of a slowly-varying background), together with two different simulations for the oscillating component of the resistance (colored lines). These simulated curves consist of a sum of cosine terms, with amplitudes and frequencies determined from the magnetic breakdown 
part of the FFT spectrum (the sum is limited to those components which are most clearly resolved, see Fig. 2b). The top panel simulation includes a $\pi$ phase for the breakdown orbits corresponding to closed loops around an odd number of petals $(A+\alpha, A+3 \alpha, \ldots)$, while the bottom panel simulation has no phase factor. The comparison between Figures $[3 \mathrm{a}$ and $3 \mathrm{~b}$ s hows that the Berry phase must be taken into account in order to correctly describe the data, thus confirming the topological nature of ZrSiS. Moreover, magnetic breakdown orbits that selectively enclose band touching points (i.e. singularities of the Berry curvature) are shown here, for the first time, to provide a novel probe of topological systems.

These collective results, together with the more detailed angular dependent study of the low frequency oscillations illustrated in Fig. S5 of the Supplementary Information, firmly establish the topological nodal loop structure in ZrSiS that was predicted in earlier band structure calculations [12, 26]. Indeed, for B $\| c$, only minor corrections to the dog-bone orbit were required to get a consistent match with all of the peaks identified in the FFT. This is the first main finding of our study. In the following, we turn our attention to the quasiparticle masses extracted from these measurements and arguably the most surprising finding, namely the anomalous temperature and field dependences of the $\mathrm{SdH}$ oscillation amplitude.

\section{MASS ENHANCEMENT ALONG THE NODAL LINE}

Figure 4 a shows a series of raw FFT spectra for both the low (left panel) and high (right panel) frequency ranges obtained from the full field sweeps performed at different temperatures between 1.5 and $60 \mathrm{~K}$. By restricting the field range however, the temperature evolution of the oscillation amplitudes was found to vary, implying that the effective masses of the quasiparticles performing each orbit were actually field-dependent. The masses obtained from such spectra are shown in Fig. $4 \mathrm{~b}$ for the petal and dog-bone orbits (see Fig. S6 of the Supplementary Information for the corresponding temperature plots). For the petal orbit, $m^{*}$ varies only slightly with field with an average value $m^{*}=0.21 \pm 0.03 m_{e}$, in reasonable agreement with previous low-field measurements [18, 19, 21] and band structure estimates of $m^{*}=0.16 \pm 0.02 m_{e}$.

For the dog-bone orbit, however, the situation is strikingly different. Firstly, $m^{*}$ is found to have a value larger than $1.0 m_{e}$ for all field ranges. This is significantly enhanced with 
respect to the band-derived value of $0.55 m_{e}$. Secondly, $m^{*}$ is found to become heavier with increasing field strength. At the highest field range, the effective mass of the dog-bone orbit is enhanced by up to a factor of 3. Such an enhancement is beyond that expected from a conventional electron-phonon interaction and implies significant polaronic or correlation effects. Indeed, this is the largest mass enhancement ever observed for a Dirac system and is particularly striking given the extremely wide $(2 \mathrm{eV})$ band width of the linear dispersion.

\section{DISCUSSION}

According to Roy and Huh et al. [16, 20], the specific properties of the band dispersions near $\epsilon_{F}$ in a NLSM, in particular the presence of the nodal line running parallel to the Fermi surface, mean that the Coulomb interaction is only partially screened due to a vanishing density of states (DOS). In graphene, with isolated Dirac points, electron-electron interactions are also only partially screened. This leads to a modification of the Dirac dispersion in such a way that the $m^{*}$ is found to shrink as $\epsilon_{F}$ approaches the Dirac point [28, 29]. In ZrSiS, the Fermi line node is not actually pinned to the Fermi level and therefore the DOS does not vanish precisely at $\epsilon_{F}$. Nevertheless, the fact that the largest mass enhancement is found for quasiparticles on the dog-bone pocket which runs parallel to the nodal loop suggests that residual Coulomb interactions lead to an enhancement in $m^{*}$ rather than a reduction. Smaller, but still finite, effects are also expected for the petal orbit. Significantly, ARPES sees no renormalization of the band dispersion [11, 12], suggesting that the renormalization only occurs very close to $\epsilon_{F}$ and is thus unobservable by ARPES.

This field-induced enhancement of $m^{*}$ for the $\beta$ pocket is reminiscent of what is observed in certain correlated electron systems such as $\mathrm{YbRh}_{2} \mathrm{Si}_{2}$ [30] or $\mathrm{CeCoIn}_{5}$ [31] that can be tuned to a quantum critical point (QCP) by a magnetic field; the approach to the QCP is reflected in a divergence of $m^{*}$ due to a dressing of the quasiparticles by the quantum fluctuations associated with the adjacent ordered state. In NLSMs, theoretical predictions for continuous quantum phase transitions into various ordered states have emerged, both in

the bulk [16] and on the surface [17], provided onsite or nearest-neighbor interactions are sufficiently strong. In ZrSiS, the additional mass enhancement in field possibly arises from the raising (through Zeeman splitting) of one of the spin sub-systems towards the Fermi level, which in turn (due to the vanishing DOS) leads to enhanced correlation effects. In 
this regard, it would certainly be interesting to explore the evolution of this enhancement to even higher fields in due course.

The final, novel finding from our study is the departure from the canonical LifshitzKosevich (LK) form of the T-dependence of the oscillation amplitudes specifically for the breakdown orbits, representative plots of which are shown in Figure 4k. While either the low- $T$ or high- $T$ data can be fitted approximately using the standard LK form [32], there is a clear discontinuity in all cases at a temperature $T_{0} \sim 8 \mathrm{~K}$. The corresponding effective mass extracted from the LK fit above $T_{0}$ is comparable to that obtained for the $\alpha$ pocket, while below $T_{0}$, it increases by approximately one order of magnitude. We are not aware of any physical origin for such a transition, particularly given that there is no concomitant change in the Fermi surface topology (i.e in the oscillation frequencies) with temperature. Phenomenologically, this type of behavior can be attributed to a boson mode with an energy of the order of $T_{0}$ that strongly renormalizes the electron effective mass only within a nonadiabatic layer $\left|\epsilon-\epsilon_{F}\right|<T_{0}$, similar to the case of electron-phonon interaction [33]. More specific assumption on the origin of this boson mode will be presented below.

The oscillation amplitude for each breakdown orbit depends not only on the effective mass associated with the orbit, but also on the probability to tunnel across the breakdown gap. Thus with increasing temperature, the magnetic breakdown may become thermallyassisted, leading to an enhancement in the tunneling probability and an upward deviation from the LK form for the oscillation amplitude. Within such a scenario, however, one might expect the deviation to be gradual [34] and not sharp as observed in ZrSiS, unless of course, the breakdown gap itself also shrinks as the temperature is increased. It is also worth noting here that no deviations from the LK formula have been reported in other breakdown systems such as organic salts or elements such as Zn.

A recent theoretical study of quantum oscillatory phenomena in gapped NLSMs predicts an anomalous $T$-dependence in the oscillation amplitude [35], though not of the form reported here for $\mathrm{ZrSiS}$. There, the amplitude is expected to show a non-monotonic dependence, collapsing to zero as $T \Rightarrow 0$ due to the presence of the hybridization gap. In $\mathrm{ZrSiS}$, of course, the small hybridization gap is bridged at high fields by the breakdown orbits and thus the non-monotonic behavior will be absent. Nevertheless, the sharp upturn in the oscillation amplitude found in ZrSiS does not appear to be consistent with such a picture.

The sharpness of the deviation is somewhat reminiscent of the behavior reported recently 
[24] in the candidate topological Kondo insulator $\mathrm{SmB}_{6}$ [36]. Despite having a bulk insulating state, quantum oscillations (in the magnetic torque) were observed below $25 \mathrm{~K}$ with multiple frequencies up to $15 \mathrm{kT}$ [24]. Below $1 \mathrm{~K}$, however a dramatic upward enhancement of the oscillation amplitude, by almost one order of magnitude, was seen in one of the low frequency orbits $(F=330 \mathrm{~T})$. The origin of such oscillations is still a subject of ongoing debate and to date, no corresponding oscillations have been seen in the electrical resistivity.

Inspection of the nodal-loop Fermi surface projection of $\mathrm{ZrSiS}$, shown in the inset of Fig. 4, suggests that the unusual mass enhancement on the breakdown orbits could be linked to enhanced, large- $Q$ density wave correlations (spin or charge) across near-nested sections of the diamond-shaped Fermi surface. In this case, one can expect a logarithmically divergent effective mass at the threshold of instability that in principle, can make the renormalization extremely strong [37]. The effective energy scale of the relevant large- $Q$ boson would have to be very low, however, in order to account for the marked change in the effective mass of the quasiparticles involved in the breakdown orbits around $T_{0}$. The field-enhanced mass found for the $\beta$ pocket, by contrast, could arise from small- $Q$ density-wave correlations, presumably across the opposite faces of the dog-bone. Such a scenario requires both short[16] and long-range [15] many-body interactions to be strongly enhanced in ZrSiS.

The field of topological semi-metals is only just emerging. The addition of correlation effects, as suggested by this present study, opens up new frontiers for the exploration of novel exotic states in these systems. Indeed, theorists have already predicted the creation of novel correlation-induced phase transitions to ordered states, both in the bulk and on the surface. Moreover, the associated quantum phase transition is believed to realize entirely new critical universality classes [17]. The work presented in this letter suggest that correlation effects are indeed strong in ZrSiS and thus, with careful manipulation of this system, e.g. through doping studies, such novel transitions associated with the topological nodal loop, at or close to half-filling, could be induced. 


\section{METHODS}

\section{Sample synthesis and characterization}

Single crystals were grown out of the elements using iodine vapour transport. Stoichiometric amounts of the elements and a small amount of iodine were placed in a carbon coated quartz tube and heated to $1100^{\circ} \mathrm{C}$ with a $100^{\circ} \mathrm{C}$ temperature gradient for 1 week. The obtained crystals were subsequently annealed at $600^{\circ} \mathrm{C}$ for a period of 4 weeks. The crystal structure was confirmed with single-crystal X-ray and electron diffraction.

\section{Magnetotransport measurements}

We studied two samples with parallel-piped shape. Six electrical contacts were defined on each sample with silver conductive paste; two of them, uniformly covering the two smaller faces of the samples, were employed as current source and drain, while the other four acted as voltage probes on different lateral sides (equivalent data were collected on the two lateral sides of the samples, indicating high homogeneity in terms of charge distribution and current flow). The resistance data were acquired in four probe configuration, with a constant current excitation of $3 \mathrm{~mA}$, and standard lock-in acquisition at $13 \mathrm{~Hz}$ frequency. The two samples were attached to a rotating stage and simultaneously characterized in a ${ }^{4} \mathrm{He}$ VTI system with base temperature $1.5 \mathrm{~K}$. The cryogenic system was accommodated in the room- $T$ access bore (32 mm diameter) of a resistive Bitter magnet at HFML, with maximum field $33 \mathrm{~T}$. The samples displayed highly symmetric resistance signals with respect to the magnetic field direction, hence only data obtained for positive magnetic field orientation are shown in the paper.

\section{Electronic structure calculations}

Electronic structure calculations were performed using a full-potential linearized augmented plane-wave plus local orbital method as implemented in the Wien2K package [38]. The experimental lattice parameters and internal positions were used $(a=3.544 \AA$, $c=$ $8.055 \AA, Z_{Z r}=0.2725, Z_{S}=0.6220$ [25]) along with the PBE-GGA exchange correlation potential [39]. The spin-orbit interaction was included using a second variational method 
[38]. The calculation was converged with $10^{4} k$-points in the full Brillouin zone. For rendering of the Fermi surface (Fig. 1 c) the energy eigenvalues were evaluated on a much more dense grid of $4 \times 10^{6} \mathrm{k}$-points in order to minimise interpolation errors which become severe as the Fermi level approaches the nodal line. In order to accurate calculate the breakdown gap, an even more dense mesh with $k$-point spacing smaller than $10^{-3} a$ was necessary.

* Electronic address: n.e.Hussey@science.ru.nl

$\dagger$ Electronic address: s.wiedmann@science.ru.nl

[1] G. E. Volovik, The universe in a helium droplet (Clarendon Press, Oxford, 2003); Lect. Notes Phys. 718, 31 (2007).

[2] C. L. Kane and E. J. Mele, $\mathrm{Z}_{2}$ topological order and the quantum spin Hall effect. Phys. Rev. Lett. 95, 146802 (2005).

[3] B. A. Bernevig, T. L. Hughes, and S.-C. Zhang, Quantum spin Hall effect and topological phase transition in HgTe quantum wells. Science 314, 1757 (2006).

[4] J. E. Moore and L. Balents, Topological invariants of time-reversal-invariant band structures. Phys. Rev. B 75, 121306 (2007).

[5] M. König, S. Wiedmann, C. Brune, A. Roth, H. Buhmann, L. Molenkamp, X.-L. Qi, and S.-C. Zhang, Quantum spin Hall insulator state in HgTe quantum wells. Science 318, 766 (2007).

[6] S. M. Young, S. Zaheer, J. C. Y. Teo, C. L. Kane, E. J. Mele and A. M. Rappe, Dirac semimetal in three dimensions. Phys. Rev. Lett. 108, 140405 (2012).

[7] S. M. Young and C. L. Kane, Dirac semimetals in two dimensions. Phys. Rev. Lett. 115, $126803(2015)$.

[8] H. Weng et al., Topological node-line semimetal in three-dimensional graphene networks. Phys. Rev. B 92, 045108, (2015).

[9] Y. Wu, L-L. Wang, E. Mun, D.D. Johnson,D. Mou, L. Huang, Y. Lee, S.L. Budko, P.C. Caneld and A. Kaminski. Dirac node arcs in $\mathrm{PtSn}_{4}$. Nature Phys. 12, 667 (2016).

[10] G. Bian, T.-R. Chang, R. Sankar, S.-Y. Xu, H. Zheng, T. Neupert, C.-K. Chiu, S.-M. Huang, G. Chang, I. Belopolski, D. S. Sanchez, M. Neupane, N. Alidoust, C. Liu, B. Wang, C.C. Lee, H.-T. Jeng, C. Zhang, Z. Yuan, S. Jia, A. Bansil, F. Chou, H. Lin and M. Zahid Hasan, Topological nodal-line fermions in spin-orbit metal $\mathrm{PbTaSe}_{2}$, Nature Commun. 7, 
$10556(2016)$.

[11] M. Neupane, I. Belopolski, M. M. Hosen, D. S. Sanchez, R. Sankar, M. Szlawska, S-Y. Xu, K. Dimitri, N. Dhakal, P. Maldonado, P. M. Oppeneer, D. Kaczorowski, F. Chou, M. Z. Hasan and T. Durakiewicz, Observation of topological nodal fermion semimetal phase in ZrSiS, Physical Review B 93, 201104(R) (2016).

[12] L. M. Schoop, M. N. Ali, C. Straßer, A. Topp, A. Varykhalov, D. Marchenko, V. Duppel, S. S.P. Parkin, B. V. Lotsch and C. R. Ast, Dirac cone protected by non-symmorphic symmetry and three-dimensional Dirac line node in ZrSiS, Nature Commun. 7, 11696 (2016).

[13] A. A. Burkov, Topological semimetals. Nature Mater. 15, 1145 (2016).

[14] C. Fang, H. Weng, Xi Dai and Z. Fang, Topological nodal line semimetals. arXiv:1609.05414v1 (2016).

[15] Y. Huh, E.-G. Moon and Y.-B. Kim, Long-range Coulomb interaction in nodal-ring semimetals. Phys. Rev. B 93, 035138 (2016).

[16] B. Roy, Interacting line-node semimetal and spontaneous symmetry breaking. arXiv:1607.07867v1 (2016).

[17] J. Liu and L. Balents, Correlation and transport phenomena in topological nodal-loop semimetals, arXiv:1609.05529v1 (2016).

[18] X. Wang, X. Pan, M. Gao, J. Yu, J. Jiang, J. Zhang, H. Zuo, M. Zhang, Z. Wei, W. Niu, Z. Xia, X. Wan, Y. Chen, F. Song, Y. Xu, B. Wang, G. Wang and R. Zhang, Adv. Electron. Mater. 2, 1600228 (2016).

[19] M. N. Ali, L. Schoop, C. Garg, J. M. Lippmann, E. Lara, B. Lotsch and S. Parkin, Buttery magnetoresistance, quasi-2D Dirac Fermi surfaces and a topological phase transition in ZrSiS. arXiv:1603.09318v2 (2016).

[20] J. Hu, Z. Tang, J. Liu, Y. Zhu, J. Wei and Z. Mao, Evidence of Dirac cones with 3D character probed by dHvA oscillations in nodal-line semimetal ZrSiS. arXiv:1604.01567 (2016).

[21] R. Singha, A. Pariari, B. Satpati and P. Mandal, Titanic magnetoresistance and signature of non-degenerate Dirac nodes in ZrSiS. arXiv:1602.01993v2 (2016).

[22] J. Hu, Z. Tang, J. Liu, X. Liu, Y. Zhu, D. Graf, K. Myhro, S. Tran, C.-N. Lau, J. Wei and Z. Mao, Evidence of topological nodal-line fermions in ZrSiSe and ZrSiTe. Phys. Rev. Lett. 117, 016602 (2016).

[23] A. Topp, J. Lippmann, A. Varykhalov, V. Duppel, B. V. Lotsch, C. R. Ast and L. M. Schoop, 
Non-symmorphic band degeneracy at the Fermi level in ZrSiTe. New J. Phys. (in press, 2016).

[24] B. S. Tan, Y.-T. Hsu, B. Zeng, M. Ciomaga Hatnean, N. Harrison, Z. Zhu, M. Hartstein, M. Kiourlappou, A. Srivastava, M. D. Johannes, T. P. Murphy, J.-H. Park, L. Balicas, G. G. Lonzarich, G. Balakrishnan and S. E. Sebastian, Unconventional Fermi surface in an insulating state, Science 349, 287 (2015).

[25] A. Klein Haneveld and F. Jellinek, Zirconium silicide and germanide chalcogenides preparation and crystal structures. Rec. Trav. Chim. Pays-Bas 83, 776 (1964).

[26] Q. Xu, Z. Song, S. Nie, H. Weng, Z. Fang and X. Dai, Two-dimensional oxide topological insulator with iron-pnictide superconductor LiFeAs structure. Phys. Rev. B 92, 205310 (2015).

[27] Y.-Y. Lv, B.-B. Zhang, X. Li, S.-H. Yao, Y. B. Chen, J. Zhou, S.-T. Zhang, M.-H. Lu and Y.-F. Chen, Extremely large and significantly anisotropic magnetoresistance in ZrSiS single crystals, Appl. Phys. Lett. 108, 244101 (2016).

[28] D. C. Elias, R. V. Gorbachev, A. S. Mayorov, S. V. Morozov, A. A. Zhukov, P. Blake, L. A. Ponomarenko, I. V. Grigorieva, K. S. Novoselov, F. Guine and A. K. Geim, Dirac cones reshaped by interaction effects in suspended graphene, Nature Phys. 7, 701 (2011).

[29] G. L. Yu, R. Jalil, B. Belle, A. S. Mayorov, P. Blake, F. Schedin, S. V. Morozov, L. A. Ponomarenko, F. Chiappini, S. Wiedmann, U. Zeitler, M. I. Katsnelson, A. K. Geim, K. S. Novoselov and D. C. Elias, Interaction phenomena in graphene seen through quantum capacitance. Proc. Natl. Acad. Sci. 110, 3282 (2013).

[30] P. Gegenwart, J. Custers, C. Geibel, K. Neumaier, T. Tayama, K. Tenya, O. Trovarelli and F. Steglich, Magnetic-field induced quantum critical point in $\mathrm{YbRh}_{2} \mathrm{Si}_{2}$. Phys. Rev. Lett. 89, $056402(2002)$.

[31] J. Paglione, M. A. Tanatar, D. G. Hawthorn, E. Boaknin, R. W. Hill, F. Ronning, M. Sutherland, L. Taillefer, C. Petrovic and P. C. Canfield, Field-induced quantum critical point in $\mathrm{CeCoIn}_{5}$. Phys. Rev. Lett. 91, 246405 (2003).

[32] D. Shoenberg, Magnetic Oscillations in Metals (Cambridge: Cambridge University Press, 1984).

[33] J. R. Schrieffer, Theory of Superconductivity (Perseus Books, 1983).

[34] K. Kishigi, M. Nakano, K .Machida and Y. Hori, dHvA effect with quantum interference oscillation due to magnetic breakdown. J. Phys. Soc. Japan 64, 3043 (1995).

[35] H. K. Pal, F. Piechon, J.-N. Fuchs, M. Goerbig and G. Montambaux, Quantum Oscillations 
in Gapped Systems. arXiv:1604.01688v2 (2016).

[36] M. Dzero, K. Sun, V. Galitski, and P. Coleman, Topological Kondo insulators. Phys. Rev. Lett. 104, 106408 (2010).

[37] A. Virosztek and J. Ruvalds, Nested Fermi-liquid theory, Phys. Rev. B 42, 4064 (1990).

[38] P. Blaha, K. Schwarz, G. K. H. Madsen, D. Kvasnicka and J. Luitz, WIEN2k, An augmented plane wave + local orbitals program for calculating crystal properties (Karlheinz Schwarz, Techn. Universitt Wien, Austria, 2001). ISBN 3-9501031-1-2.

[39] J. P. Perdew, S. Burke and M. Ernzerhof, Generalized gradient approximation made simple. Phys. Rev. Lett. 77, 3865 (1996).

\section{Acknowledgements}

We acknowledge enlightening discussions with Y.-B. Kim and A. McCollam. We also acknowledge the support of the HFML-RU/FOM, member of the European Magnetic Field Laboratory (EMFL). A portion of this work was supported by the Engineering and Physical Sciences Research Council (grant no. EP/K016709/1).

\section{Author contributions}

S.W. initiated the project in collaboration with L.S.. S.P., M.v.D. and S.W. performed the magnetotransport measurements. L.S. and B.L. synthesized the ZrSiS single crystals. A.C. performed the electronic band structure calculations. S.P., M.v.D., S.W., A.C., M.I.K. and N.E.H. analysed the data. N.E.H. wrote the manuscript with input from all the coauthors.

\section{Materials and correspondence}

Correspondence and requests for materials should be addressed to S.W. (s.wiedmann@science.ru.nl) and N.E.H. (n.e.hussey@science.ru.nl). 
A

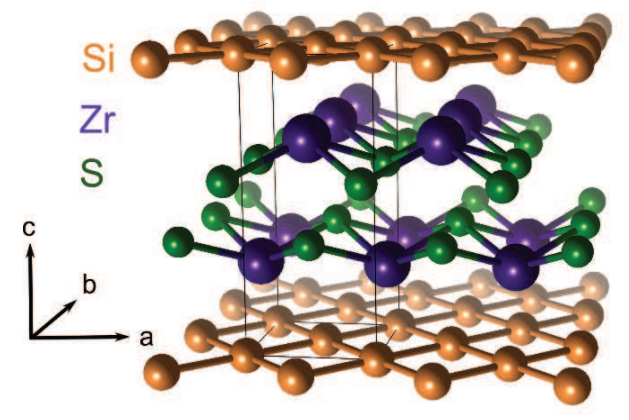

B

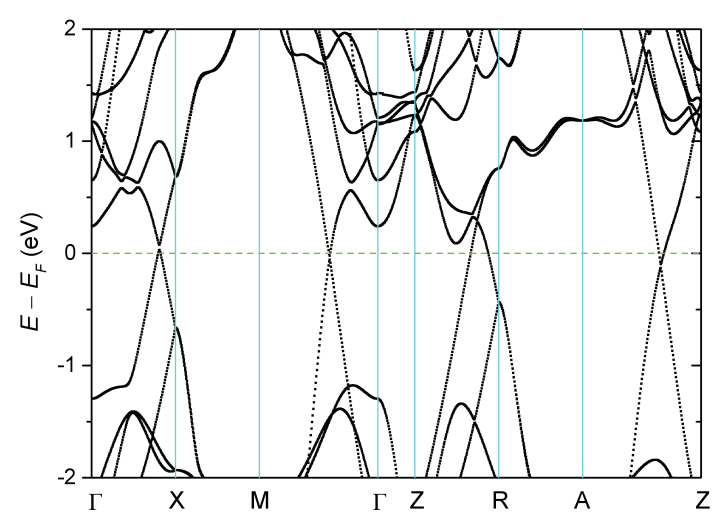

C

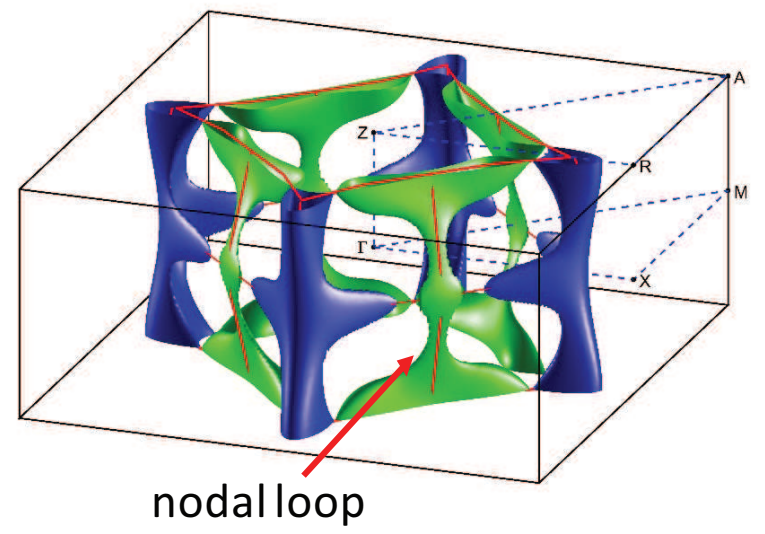

FIG. 1: 
A
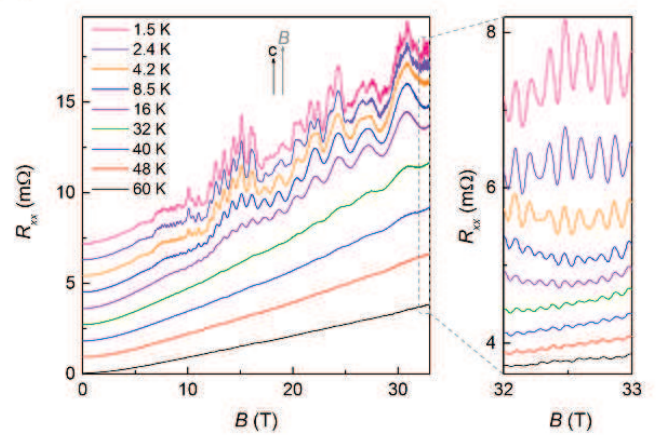

B

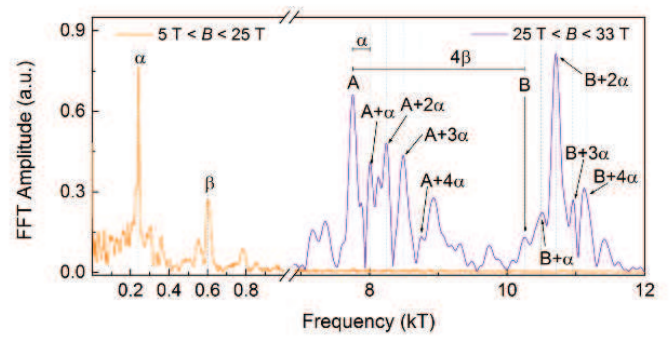

c

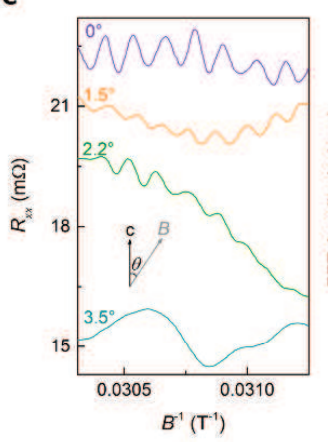

D

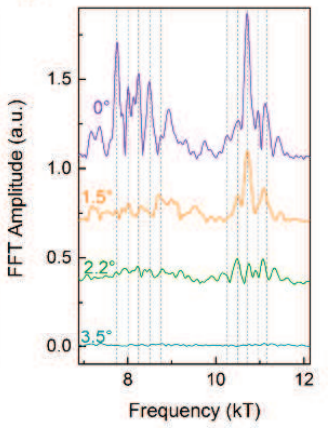

E
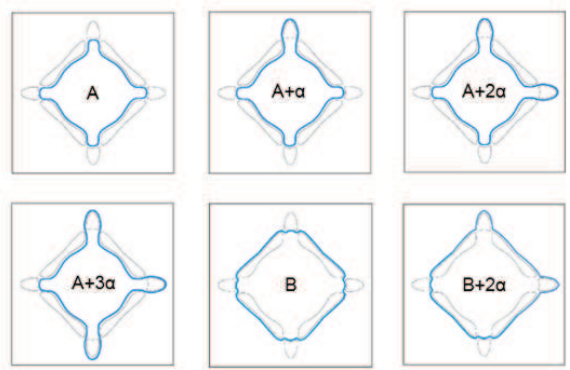

F

G
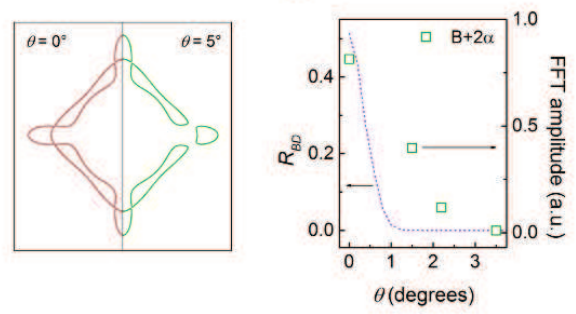

FIG. 2: 


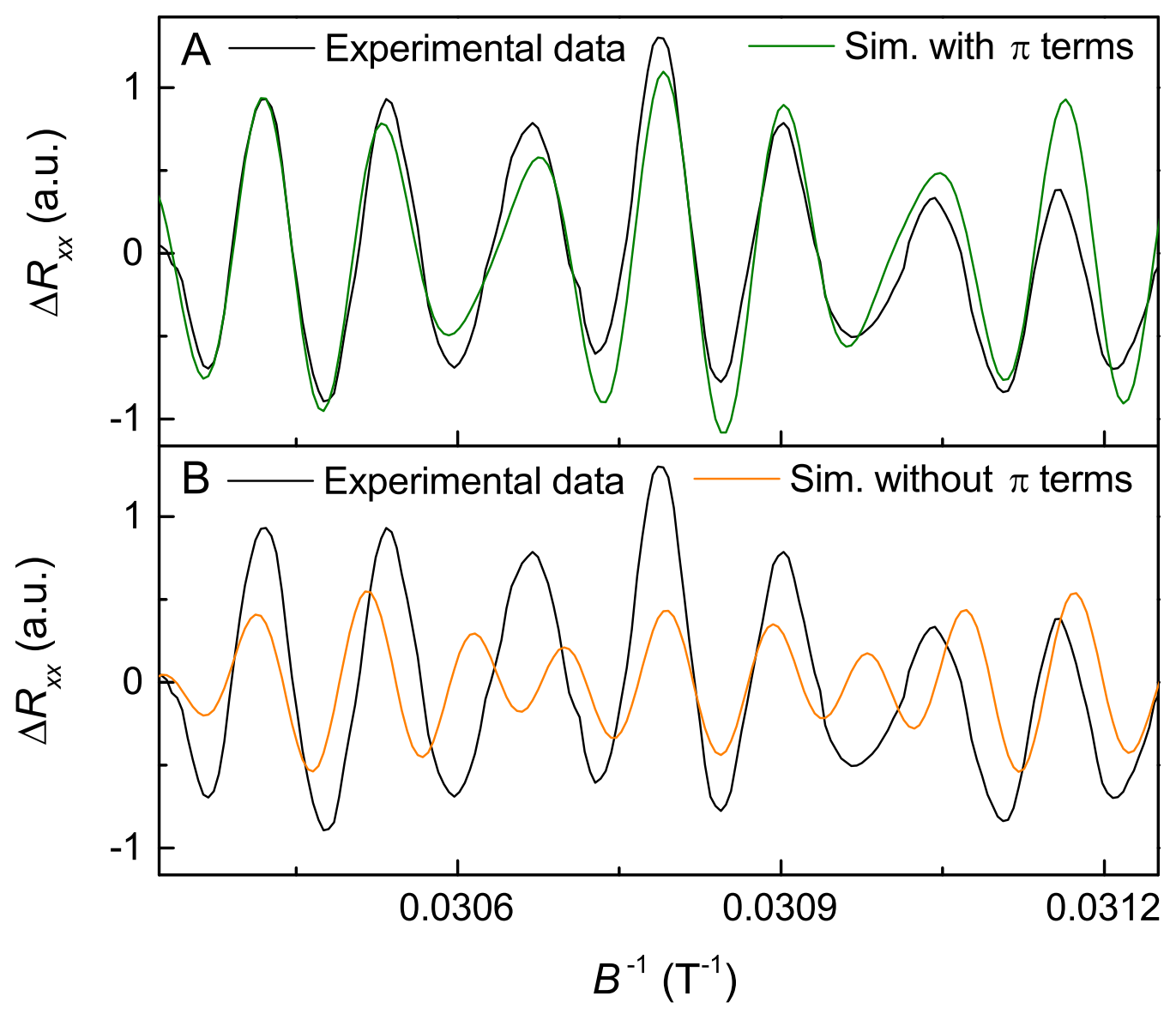

FIG. 3: 

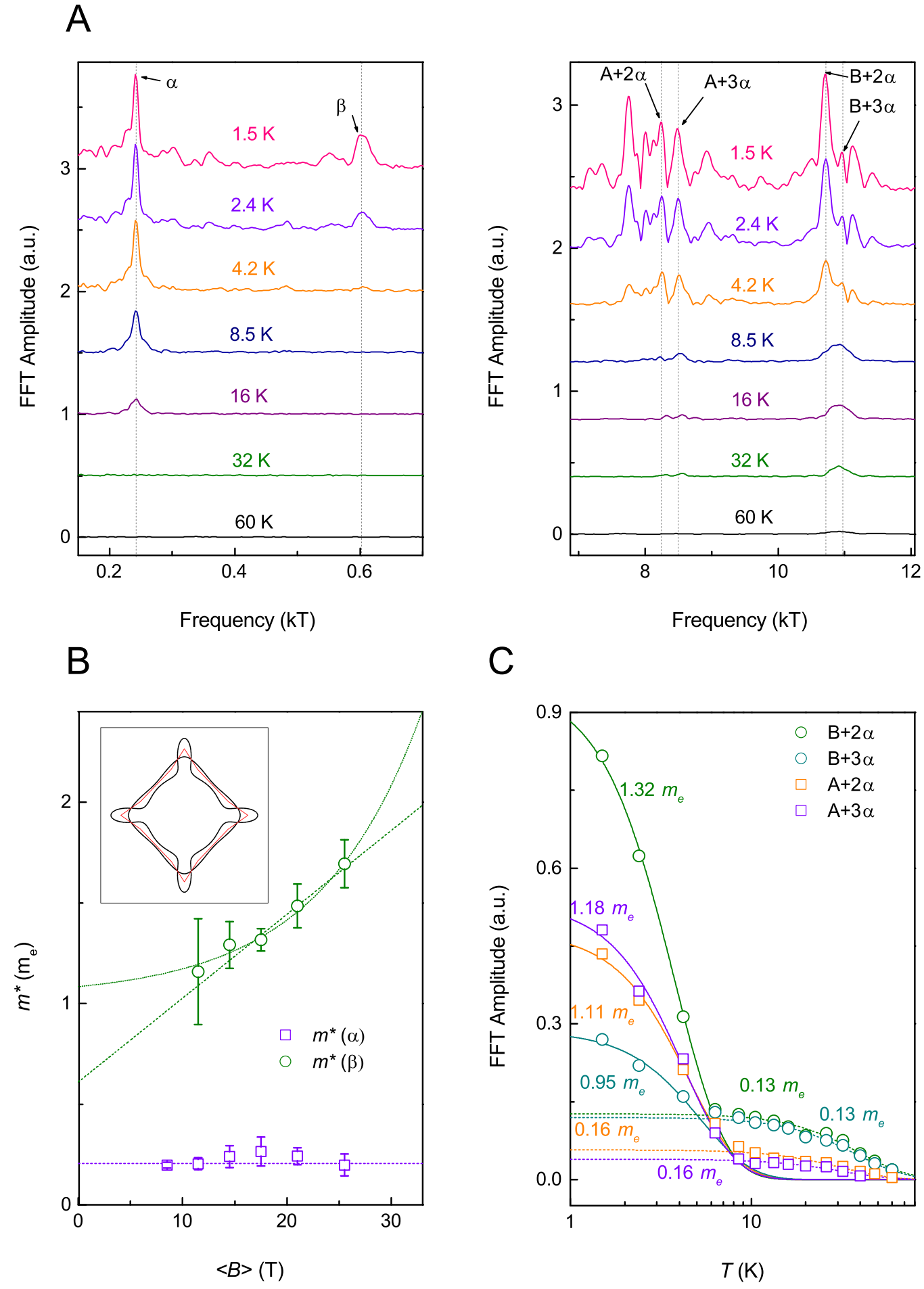

FIG. 4: 


\section{FIGURE CAPTIONS}

Figure 1: Crystallographic and electronic structure of ZrSiS. (a) Crystal structure of ZrSiS. The Si square net can be seen in blue. (b) Calculated bulk band structure with spin-orbit coupling. (c) Resultant three-dimensional Fermi surface. The top surface of the Brillouin zone contains the symmetry points Z, R and A. The location of the Dirac nodal loop is shown by the dashed black line.

Figure 2: Shubnikov-de Haas oscillations and breakdown orbits in ZrSiS in a perpendicular magnetic field (a) Left panel: Series of magnetoresistance (MR) sweeps up to $33 \mathrm{~T}$ with $\mathbf{B} \| c$ at the temperatures listed (curves are offset for clarity). Right panel: Close-up of the high-field part of the sweep, highlighting the fast oscillations that are associated with the onset of magnetic breakdown. (b) Fast Fourier transform (FFT) of the full MR sweep, having subtracted off a smooth polynomial background fit. The spectrum is divided into low a frequency part encompassing the frequencies due to the $\alpha$ (petal) pocket $(F=240 \mathrm{~T})$ and $\beta$ (dog-bone) pocket $(F=600 \mathrm{~T})$ and a high frequency part that includes the frequencies of the breakdown orbits. The magnetic field ranges considered for the two parts are indicated in the figure. Label $A$ corresponds to the 'inner' breakdown orbits, $B$ to the 'outer' orbits as illustrated in Fig. 2(e). (c) Polar angle dependence of the high-field MR sweeps as a function of $1 / B$ and $(d)$ corresponding FFT spectra, showing the strong suppression of the high frequency (breakdown) oscillations with tilt angle. (e) In-plane projection of the diamond Fermi surface at $\theta=0^{\circ}$; the outer square represents the first Brillouin zone. Several of the breakdown orbits are indicated, their labels refer to the corresponding peaks in the FFT spectrum. (f) In-plane projection of the diamond Fermi surface for $\theta=0^{\circ}$ (left panel) and $\theta=5^{\circ}$ (right panel). In a tilted field, certain breakdown gaps increase in magnitude. (g) Attenuation of the oscillation amplitude as a function of $\theta$. The solid line is a calculation of the damping term. See the Supplementary Information for more details.

Figure 3: Evidence for the topological character of the nodal loop. (a), (b) Black line: Oscillatory part of the magnetoresistance trace at $1.5 \mathrm{~K}(\mathbf{B} \| c)$, as a function of $1 / B$, between $32 \mathrm{~T}$ and $33 \mathrm{~T}$. The green curve in (a) corresponds to 
$\sum_{n} a_{n} \cos \left(2 \pi F_{A, n} / B+n \pi\right)+b_{n} \cos \left(2 \pi F_{B, n} / B+n \pi\right)$, i.e. a sum of cosine functions with amplitudes $\left(a_{n}\right.$ and $\left.b_{n}\right)$ and frequencies $\left(F_{A, n}\right.$ and $\left.F_{B, n}\right)$ given by the main peaks of the FFT in the right part of Figure $2 \mathrm{~b}$, and including a non-trivial Berry phase term $(n \pi)$ for the breakdown orbits encircling an odd numbers of band-touching vertexes. The orange trace in (b) is the same function of the green one in (a), without the $n \pi$ terms.

Figure 4: Effective mass and anomalous thermal damping of the oscillation amplitude. (a) Fast Fourier transforms of the full magnetoresistance sweeps performed with B $\| c$ at the different temperatures listed for both the (left panel) low-frequency and (right panel) high-frequency oscillations (same magnetic field ranges of Fig 2 $\mathrm{b}$ ). (b) Effective masses for the $\alpha$ and $\beta$ pockets obtained from $T$-dependence of the oscillation amplitudes over the different field ranges as indicated. The dashed and dashed-dotted lines are guides to the eye, under the assumption that the zero-field mass is unrenormalized or renormalized respectively. Inset: location of the nodal line (dashed line) within the in-plane projection of the diamond Fermi surface for $\mathbf{B} \| c$. (c) Mass plots for a number of frequencies corresponding to breakdown orbits. The solid and dashed lines are fits to the LK expression below and above $T_{0}=8 \mathrm{~K}$ respectively. Again, the quasiparticle masses extracted from each fit are given in the Figure. 